Supporting information:

\title{
Sensitive Detection of Ambient Formaldehyde by Incoherent Broadband Cavity Enhanced Absorption Spectroscopy
}

Jingwei Liu ${ }^{1,2}$, Xin Li1 ${ }^{1,2,3, *}$, Yiming Yang ${ }^{1,2}$, Haichao Wang ${ }^{1}$, Cailing Kuang ${ }^{1}$, Yuan $\mathrm{Zhu}^{1}$, Mindong Chen ${ }^{3}$, Jianlin $\mathrm{Hu}^{3}$, Limin Zeng ${ }^{1,2}$, and Yuanhang Zhang ${ }^{1,2}$

${ }^{1}$ State Key Joint Laboratory of Environmental Simulation and Pollution Control, College of Environmental Sciences and Engineering, Peking University, Beijing, 100871, China.

${ }^{2}$ International Joint Laboratory for Regional Pollution Control, Ministry of Education, Beijing, 100816, China

${ }^{3}$ Collaborative Innovation Centre of Atmospheric Environment and Equipment Technology, Nanjing University of Information Science \& Technology, Nanjing, 210044, China P. R. *Corresponding author: Xin Li (1i_xin@pku.edu.cn)

\section{Contents}

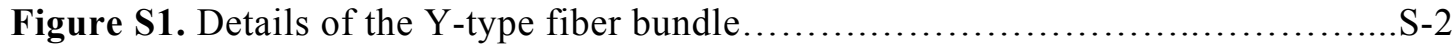

Figure S2. Transmissions of three optical filters ......................................

Figure S3. HCHO concentrations retrieved from two fit ranges......................S-4

Figure S4. Convoluted absorption cross sections of typical UV gas absorbers..............S-5

Figure S5. Continuous measurement results of reference spectra with $30 \mathrm{~s}$ time resolu-

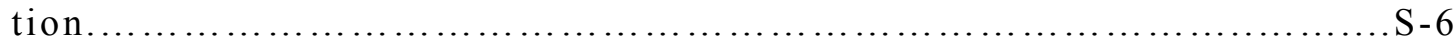

Figure S6. Regression plot of binned HCHO IBBCEAS measurements against the Hantzsch measurements 
(a)

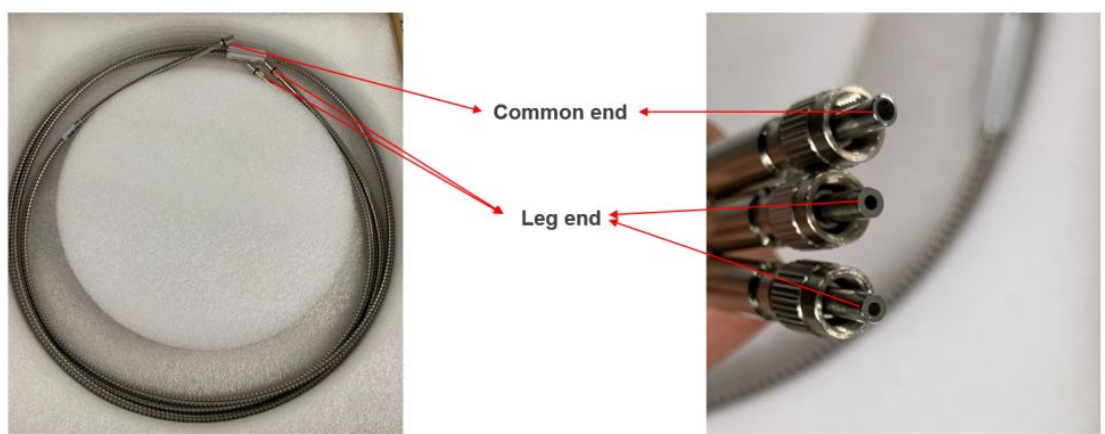

(b)

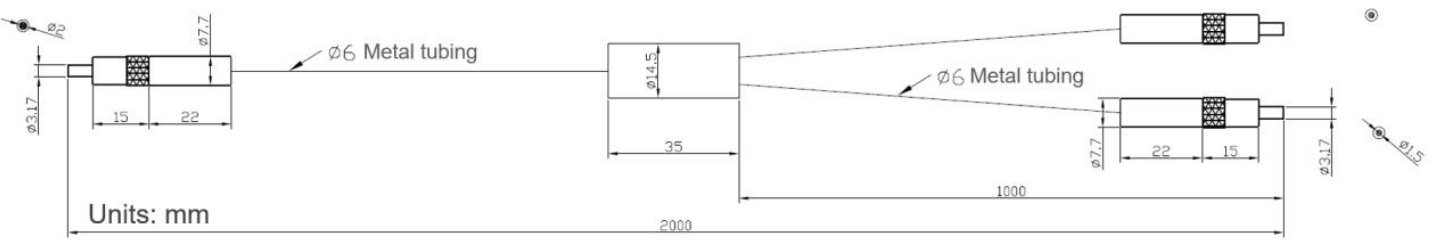

Figure S1. (a) Pictures of the Y-type fiber bundle; (b) Detailed size of the fiber bundle 


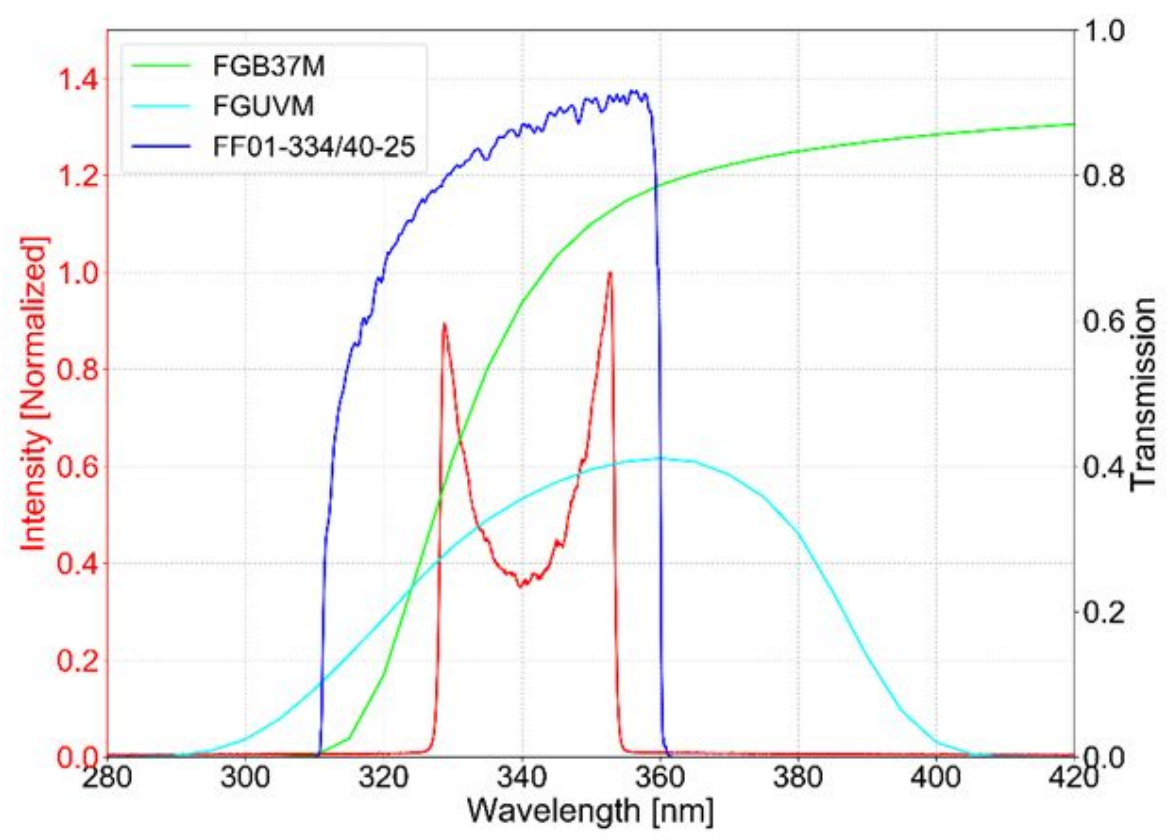

Figure S2. Transmissions of three filters (FGB37M, FGUVM, and FF01-334/40-25) provided by manufacturers. 


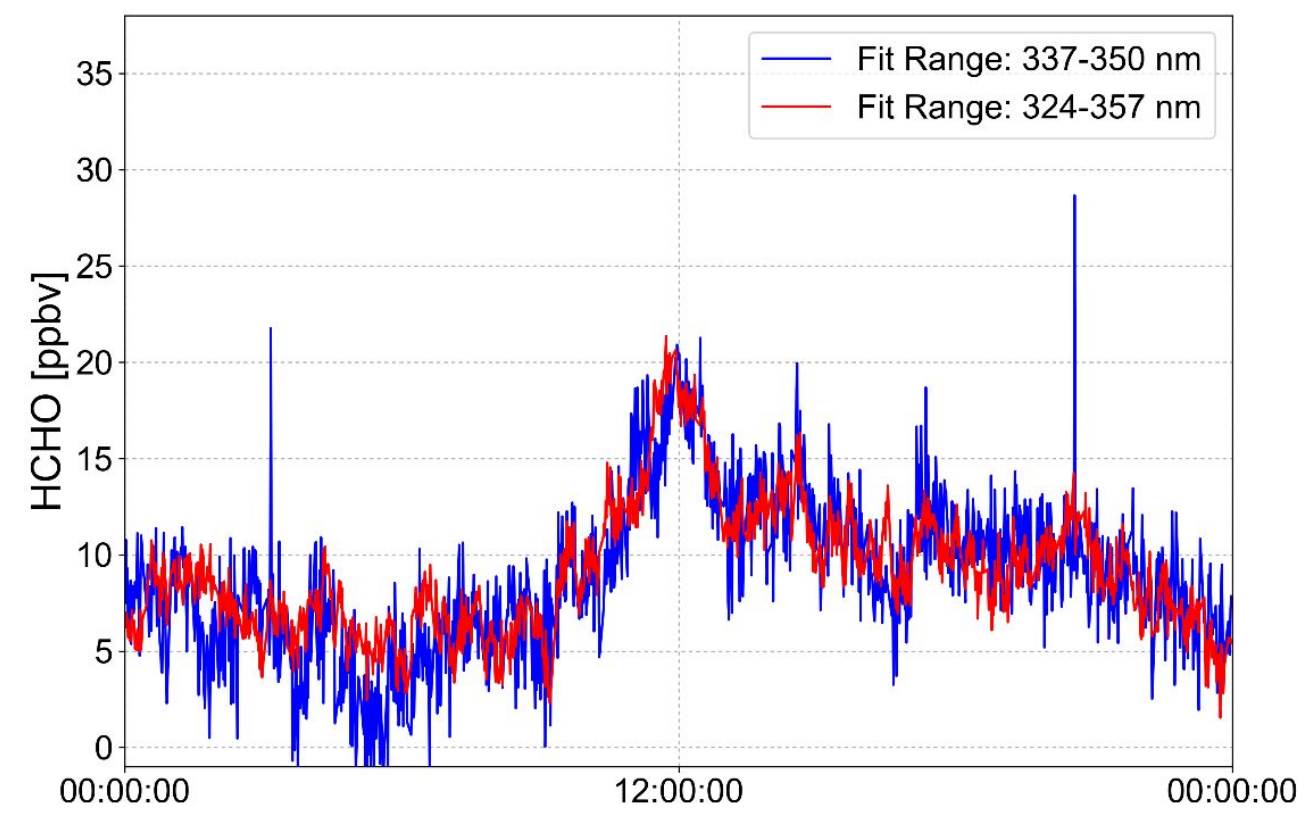

Figure S3. HCHO concentrations retrieved from two fit ranges. 


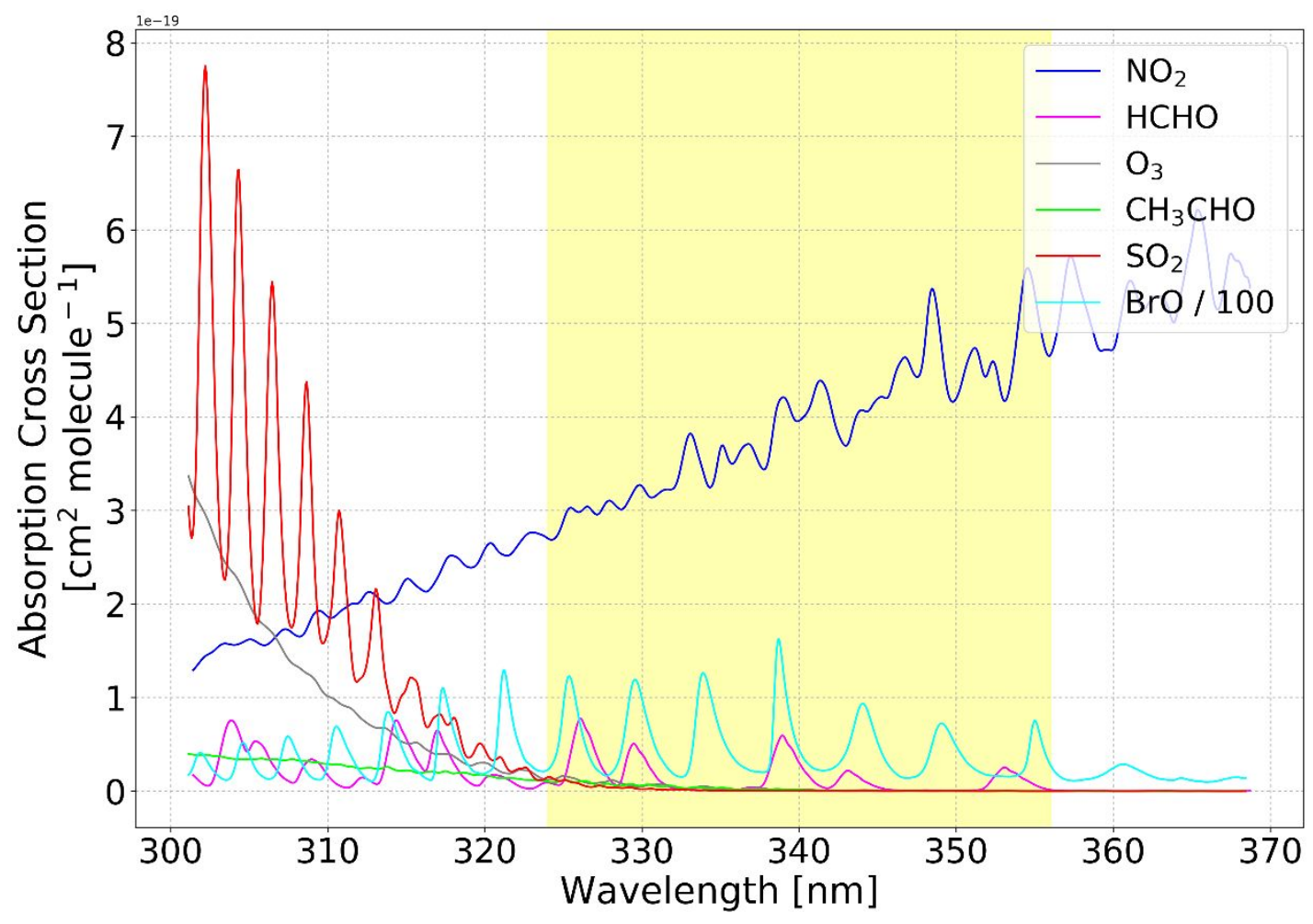

Figure S4. Convoluted absorption cross sections of typical UV gas absorbers. The BrO data were reduced by 100 -fold for clarity, and yellow overlay in the figure represented the spectral fit range. 

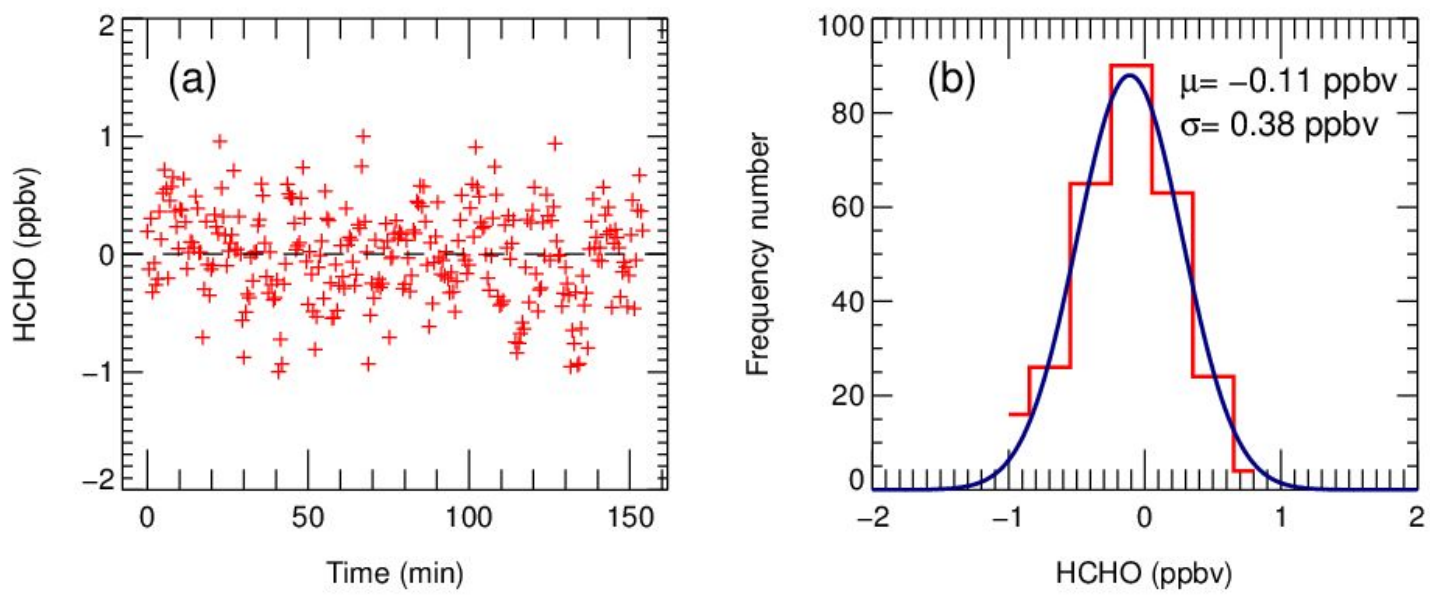

Figure S5. Continuous measurement results of reference spectra with $30 \mathrm{~s}$ time resolution: (a) time series; (b) histogram of spectral fitting results. 

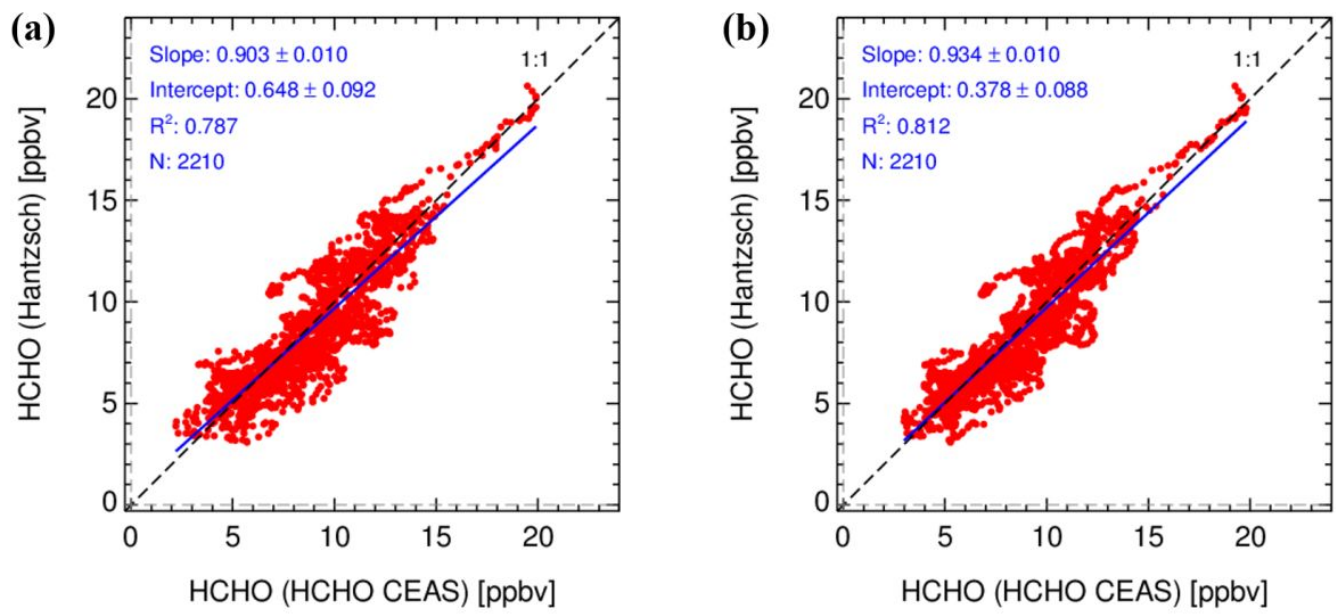

Figure S6. Regression plot of binned HCHO IBBCEAS measurements against the Hantzsch measurements: (a) 5 min smoothing; (b) 10 min smoothing. 